\title{
Filigrane
}

Écoutes psychanalytiques

\section{Aux alentours de la violence}

\section{Emmanuel Piché}

Volume 25, numéro 1, printemps 2016

Actes du colloque Le sujet sacrifié (Ghyslain Lévy)

URI : https://id.erudit.org/iderudit/1037376ar

DOI : https://doi.org/10.7202/1037376ar

Aller au sommaire du numéro

Éditeur(s)

Revue Santé mentale au Québec

ISSN

1192-1412 (imprimé)

1911-4656 (numérique)

Découvrir la revue

Citer cet article

Piché, E. (2016). Aux alentours de la violence. Filigrane, 25(1), 93-99.

https://doi.org/10.7202/1037376ar d'utilisation que vous pouvez consulter en ligne.

https://apropos.erudit.org/fr/usagers/politique-dutilisation/ 


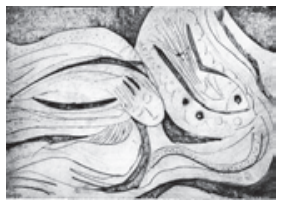

\section{Aux alentours de la violence}

\section{Emmanuel Piché}

A

lexandre L'Archevêque et Élise Bourgeois-Guérin ont ouvert plusieurs voies de réflexion sur le chemin complexe de la violence, surtout dans la prise en compte qu'à ce sujet, l'individuel et le collectif se trouvent subtilement noués. Réfléchir à la violence dans cet entrelacement est un travail à la fois difficile, troublant et nécessaire; il nous ouvre aux fondements de notre humanité indubitablement sociale, incluant sa partie «inhumaine».

J'avoue avoir accepté de discuter leur texte en reconnaissant du même coup avoir peu réfléchi spécifiquement à la question de la violence, surtout lorsqu'il s'agit de la penser dans ce qui relie ensemble l'expérience individuelle et collective. Personnellement, il m'a fallu un certain temps pour reconnaître tout l'intérêt de cette dimension dans la métapsychologie et mesurer l'ampleur de sa présence dans la clinique, notamment ce que nous avons coutume d'appeler l'aspect économique de la topique psychique. J'imagine que pour certains, dont je fus, l'œuvre d'Éros est plus facile et moins dérangeante à repérer que celle de Thanatos, surtout lorsqu'on découvre en soi, en l'autre et autour de soi l'étendue des forces et la multiplicité des visages de la destructivité humaine. S’exprimer ainsi constitue en fait une méprise, puisque l'on sait l'intrication incessante entre les différents mouvements qui animent l'âme humaine, son fonctionnement essentiellement conflictuel, sans compter que la violence du pulsionnel se trouve autant du côté de ses forces de liaison que de celles de déliaison.

Il me faudra aussi limiter et circonscrire mon commentaire en découpant dans leur texte ce qui a su attirer plus fortement mon attention qui, elle, aurait pris plaisir à s'égarer plus longuement sur diverses voies parallèles, voire sur des chemins plus déliés.

Notons d'abord que ce qui m'est venu à l'esprit dans un premier temps est la confusion qui nous gagne lorsque nous parlons de violence. De quoi s'agit-il? Agressivité, haine, sadisme, pulsion de mort, cruauté, domination, masochisme, emprise, destructivité: la liste n'est pas exhaustive des termes s'y rattachant, les distinctions et les recoupements sont nombreux. Freud 
semble avoir considérablement évolué dans sa pensée sur l'agressivité et sa théorisation de la pulsion de mort a permis d'organiser sa conception d'une dimension radicale dans l'être humain en lien avec la violence, même si ce terme est peu utilisé par lui. Je ne m'attarderai pas ici à des définitions, si ce n'est pour souligner que le terme de violence renvoie d'abord à l'idée de force, de brutalité, d'intensité, voire de démesure, à la dimension économique donc, et qu'en lui accolant l'adjectif destructrice, nous imprimons déjà un vecteur à notre réflexion. D'emblée, une question se pose: la violence est-elle en elle-même destructrice?

Par ailleurs, comme je le soulignais plus haut, en tant que clinicien d'orientation psychanalytique, la difficulté à penser à la fois l'individuel, qui nous est plus familier, et le collectif, m’apparaît un défi de grande ampleur où les maladresses et les impairs sont faciles à commettre. Est-ce qu'il faudra parler de résonances entre les deux scènes, d'effets mutuels de l'une sur l'autre, d'antériorité de l'une ou l'autre, d'emboîtement? L'ensemble des textes du colloque nous donnent la mesure de la complexité ainsi que des subtilités engagées dans cette aventure réflexive.

Finalement, toujours en avant-propos, je voulais souligner que la violence se trouve spontanément associée dans nos esprits à un «en-trop», à une quantité ou une force en surcharge qui se déverse en créant des dommages, mais que cet en-trop est par ailleurs souvent un «trop-peu», comme notre clinique nous donne régulièrement l'occasion de le constater. Troppeu et en-trop sont des catégories qui semblent aller de pair, si ce n'est qu'en ce que le trop-peu d'accompagnement et d'élaboration psychique nous laisse aux prises avec un en-trop pulsionnel originaire.

L'une des réflexions proposée par A. L'Archevêque et É. BourgeoisGuérin concerne le lien entre le travail psychothérapeutique et le champ socioculturel, en ce qui a trait à l'influence de la technologie et des réseaux sociaux sur les échanges au sein du dispositif clinique. Les auteurs allèguent une difficulté à soutenir le face-à-face du cadre psychothérapeutique et son angoisse quand les modes de communication contemporaine vont dans le sens de la brièveté des interactions, la parcellisation du propos, la possibilité de rompre les liens discrètement et d'utiliser des identités virtuelles.

Cette piste de réflexion renvoie entre autres à la question de l'identité, ici abordée en tentant de circonscrire ce qu'il en est du sujet et de ses frontières. Les nouvelles technologies, bien que nous ayons encore peu de recul sur les bouleversements et les changements qu'elles engendrent, modifient le rapport que les utilisateurs entretiennent avec eux-mêmes et avec les autres. 
Est-ce que nous pouvons penser que les transformations au sein de l'identité du sujet contemporain inscrit dans les nouvelles technologies et les réseaux sociaux favorisent l'illusion de pouvoir faire l'économie de l'autre dans l'accession à son désir et à l'objet de ce désir? Illusion également face à la possibilité de faire l'économie de l'expérience du conflit et de la perte lorsque l'autre est tenu à distance et maitrisé dans les formes et les occurrences de sa présence? Quelle est la part d'emprise impliquée à ce titre dans les nouvelles technologies et que vise-t-elle? Je me demande alors si l'engagement psychothérapeutique risque de provoquer chez les sujets contemporains des défenses plus particulièrement narcissiques, avec une menace de type persécutoire, ou si au contraire il sera l'occasion d'une trame relationnelle porteuse d'espoir, d'un espace transférentiel pour élaborer ce qu'il en est de son désir et de son identité.

Une question se dégage de ce nouveau paysage: quel type d'angoisse et quel mode de relation d'objet sont plus particulièrement mobilisés chez le sujet contemporain par le dispositif psychothérapeutique, et de quelles manières les nouvelles technologies à la fois provoquent et cherchent à remédier à cette angoisse?

Toujours concernant le thème du sujet et de ses frontières, et pour demeurer dans le sujet de la violence, les auteurs nous invitent à réfléchir à l'altérité qui implique, selon leurs mots, «altération de soi et de l'autre». Michel De M'Uzan a notamment écrit de belles choses sur la rencontre troublante des inconscients durant les séances d'analyse en créant son concept original de chimère. En cette matière, le jeu des identités impliquées représente certains risques, ce qui m'amène à poser cette question: qu'en est-il de la présence à l'autre en clinique, de la sensibilité à sa souffrance lorsqu'une quantité trop grande d'affects sans représentation est impliquée, brouillant les frontières entre soi et l'autre, et entre soi et soi si l'on peut s'exprimer ainsi, en pensant à la distance suffisante pour la mise en pensée, pour l'organisation d'une parole qui ne soit pas acte et décharge sur l'autre. La constitution d'un sujet, d'une frontière implique qu'il n'y ait pas trop d'empiétement (Winnicott) de la part de l'environnement du sujet en devenir. L'effet de la violence, celle du pulsionnel effractant des parents notamment (Ferenczi), se situe justement dans cette altération trop grande du sujet qui lui rendra plus ou moins difficile d'ériger des frontières protectrices garantes d'un rapport favorable à l'autre, difficulté potentielle également d'ériger les frontières structurantes du refoulement, ce qui risque alors de se transformer en ce double impératif meurtrier et incestueux: soi ou l'autre, soi dans l'autre. En tant que clinicien, 
comment ne pas ajouter alors à la violence du pulsionnel lorsque nous tentons de nous mettre à la place de l'autre par une empathie plus ou moins probable qui risque de plaquer sur lui ce qui servirait finalement davantage à se rassurer soi-même? Comment se laisser plutôt altérer par l'autre en séance en demeurant bien vivant?

Une autre élaboration des auteurs concerne les violences possibles de la demande du patient et de l'interprétation du clinicien. De la part du patient est perçue cette violence dans une forme de supplique à enlever la part souffrante ou défectueuse de son être et de greffer la part ou la faculté manquante, demande influencée par l'approche biomédicale. Du côté du clinicien, le danger de "presser» le patient à intégrer et à assumer la part souffrante de son être lorsque celle-ci est vécue comme un organe cancéreux qui menace sa survie, ce qui fait intervenir une éthique de la mentalisation à tout prix.

Cette élaboration nous conduit au plus près du travail clinique avec le clivage et le déni. Je ne m'attarderai pas aux considérations sur l'approche biomédicale (pharmaceutique et DSM) et sa logique additive/soustractive des souffrances humaines, approche s'inscrivant dans un contexte social qui influence assurément l'offre et la demande dans les thérapeutiques psychologiques. Non plus sur tout l'intéressant développement historique concernant la conception de l'esprit-matière, si ce n'est que pour souligner à nouveau avec vous l'omniprésence du corps dans la culture nord-occidentale actuelle et la place réduite laissée à la vie d'âme, à la dimension psychique qui est de toutes sortes de manières mise en violence. Je reprendrai donc la discussion à partir des thèmes du clivage et du déni.

La réflexion clinique autour du travail avec les angoisses primitives et les défenses archaïques a pris de l'ampleur dans les dernières décennies, ce dont témoigne l'œuvre de plusieurs auteurs psychanalytiques contemporains, dont celle majeure d'André Green. Une question centrale se pose: comment travailler avec un patient qui a désubjectivé une partie de son être, de sa personnalité, de son fonctionnement, qui s'est dissocié d'une partie de son expérience personnelle pour survivre à des angoisses de néantisation, de catastrophe, d'effondrement, d'agonie? Dans la clinique de ce qu'on pourrait appeler «les états traumatiques", là où l'on rencontre l'impact des traces agissantes d'un vécu qui se situe hors psyché, comment accompagner le patient dans l'appropriation de ce qui semble menacer sa survie psychique? Une approche psychanalytique «classique» centrée exclusivement sur l'interprétation constituerait assurément une violence faite au sujet, puisque la 
défense de celui-ci consiste justement ici en la mise hors sens et la décharge de ce qui agit en lui comme un corps étranger. Certains fonctionnements extrêmes semblent exclusivement axés sur la destruction de tout ce qui serait de l'ordre d'une subjectivation, de la psychisation, de tout processus d'humanisation; je pense ici notamment aux criminels meurtriers, violeurs, pédophiles et autres dont le psychanalyste Claude Balier a si bien illustré la dynamique d'anéantissement/toute-puissance qui gouverne leurs actes. Alors, quel type de présence, à soi et à l'autre, sera nécessaire à l'élaboration de la destructivité; quelle sera notre disponibilité pour accueillir la douleur et les angoisses au-delà et en-deçà des mots du sujet, à travers l'acte et la sensorialité, afin de l'aider à contenir et à s'approprier ce qui l'agit dans des mouvements internes dotés d'une quantité d'énergie difficile à lier, qui fait effraction et menace, qui terrifie? Est-ce que cela peut se faire sans un réel engagement du clinicien dans le «partage» de ce vécu terrorisant, partage qui implique l'acceptation des silences, de la sidération passagère, du peu de mots pour dire, de la présence du corps et de ses actes pour tenter de signifier l'irreprésentable? Et sur la scène collective, comment arriver à penser et à élaborer la violence omniprésente qui nous entoure, diffusée sans arrêt par les médias dans un débordement d'images, sans succomber à son effet anesthésiant ou fascinant, hypnotisant, et sans épouser la fuite de l'indifférence?

É. Bourgeois-Guérin et A. L'Archevêque abordent de front la question de la violence lorsque, évoquant les pulsions de vie et de mort, ils réfléchissent sur la violence pour et contre le sujet. Les exemples de la valeur adaptative de la violence (légitime défense, médecine, développement psychosexuel) ne sont pas sans rappeler les travaux de Bergeret sur la violence fondamentale, comparativement à celle qui s'exprime contre le sujet et qui serait destructrice: agressions verbales et physiques, automutilation, suicide, meurtre et guerre, acte de coupure et de déliaison, pouvoir exercé sur l'autre pour le réduire au rang d'objet, dans l'omnipotence. Ainsi se pose la question essentielle des destins possibles de notre destructivité, notamment les salvatrices sublimations qui ne représenteraient pas seulement un déplacement de but, comme le rappellent les auteurs, mais qui consisteraient dans une activité pulsionnelle désexualisée promouvant l'abaissement des tensions et l'individuation psychique. Ils évoquent cependant la possibilité que l'art se déploie également comme potentiel destructeur (scarification, fétichisation) et mettent en tension la sublimation et le passage à l'acte, ce dernier se manifestant comme décharge et court-circuitage de la vie psychique et de ses possibilités d'élaboration. 
J'aurais aimé pouvoir m'attarder à la question de la sublimation et de l'art, qui a été également abordée par Ellen Corin, mais le terrain m'apparaît si vaste et l'espace, restreint, surtout si on la met en lien avec ce que Nathalie Zaltzman a nommé travail de culture. Il serait toutefois intéressant d'interroger davantage le potentiel destructeur de l'art, qui me semble logé notamment dans l'aliénation que sa pratique provoque chez l'artiste lui-même et l'effet séducteur, analgésiant ou surexcitant, imprimé chez le destinataire. La question de la désexualisation des pulsions est également un sujet fort complexe que l'on pourrait élaborer. Le champ de réflexion reste ouvert et fort étendu dans la pratique clinique entre le pôle de la sublimation et celui du passage à l'acte.

Freud nous a invités à penser la pulsion comme orientée vers la tendance à l'actualisation, à la décharge, appelant le travail psychique afin d'endiguer sa violence, celle-ci se situant dans le sexuel infantile traversant l'activité pulsionnelle. Ce sexuel infantile a ceci de radical qu'il ne veut rien savoir de l'autre et qu'il résiste à tout effort de civilisation. Freud a conçu peu à peu qu'une partie de l'activité pulsionnelle allait dans le sens de la répétition, d'une tendance au retour à un état inorganique, tendance finalement à la déliaison et à la destruction, ce qu'il a définitivement postulé à partir de 1920 comme une pulsion de mort au cœur de l'être humain. On sait toute la controverse autour de cette notion, notamment à savoir si la destructivité est originaire et constitutive du bagage héréditaire de l'être humain ou si elle serait davantage une réponse aux défaillances de l'environnement. Cette controverse pose entre autres pour moi la question de l'aversion que nous avons à admettre cette dimension de l'être humain, en nous et autour de nous, ainsi que le scandale de sa jouissance.

S'il n'est pas donné à beaucoup de cliniciens de travailler avec des criminels violents, là où la pulsion de mort et l'agression destructrice sont plus apparentes et opérantes dans leur déflection comportementale envers l'objet, nous pourrions dire tout de même que tout un chacun exerce une violence contre l'autre, plus ou moins destructrice, violence qui peut être subtile et s'exprimer par différentes formes de déni de l'altérité de l'autre, de désir de domination, ainsi que par différentes formes d'indifférence. Par ailleurs, il nous est davantage donné de travailler avec des sujets exerçant une violence contre eux-mêmes, là où la pulsion de mort suit son courant premier d'autodestruction, dans les ratées plus ou moins grandes d'un masochisme érogène primaire qui serait protecteur de la vie psychique en reprenant à son compte ce mouvement auto pour en faire du psychique et du sexuel. Je 
pense particulièrement aux patients dépressifs et leurs mouvements mélancoliques, à la vaste clinique des masochismes et des culpabilités mortifères, à celle de l'addiction et des conduites à risque, et finalement aux patients qui somatisent. La réaction thérapeutique négative s'avère un constat de ce qu'il en est de la pulsion de mort dans le quotidien de notre clinique.

Dans ma pratique personnelle et celle de certains collègues, je demeure stupéfait de constater qu'à l'inverse de ce que l'on peut observer dans le reflet des différents médias, nos patients cherchent d'abord à se nuire à euxmêmes de toutes sortes de manières et avec une volonté parfois féroce. Ces patients nous placent devant leur dilemme terrible où ils semblent avoir choisi de se sacrifier dans le but de protéger l'autre. Est-ce que la violence de la supplique du patient dont vous parlez dans votre texte irait dans le sens de ce choix quasi automutilatoire?

Vient le temps de conclure ce commentaire qui, je m'en aperçois, tire davantage le sujet de la violence du côté de la clinique de l'individuel. Peutêtre aurait-il été possible de retisser la discussion un peu plus vers les rives du collectif, à cette intersection qui fut une part importante de la réflexion freudienne. A. L'Archevêque et É. Bourgeois-Guérin auront soulevé de, nombreuses pistes de réflexion; ils se sont engagés à mettre en mots quelque chose autour de la violence; une violence qui tend justement à nous tenir au silence, dans notre mépris et notre dégoût, je dirais organique, à considérer cette dimension destructrice de nous-mêmes, surtout dans ce qu'elle comporte de jouissance silencieuse. Là repose assurément sa plus grande menace.

Emmanuel Piché emmanuelpiche@videotron.ca 